\title{
EL TRIPLE ESTÁNDAR DE LA RAZÓN PÚBLICA
}

MoISÉS VACA

Instituto de Investigaciones Filosóficas Universidad Nacional Autónoma de México moisesvacap@gmail.com

ITZEL MAYANS

Facultad de Filosofía y Letras Universidad Nacional Autónoma de México itzel.mayans@gmail.com

RESUMEN: Varios autores afines al proyecto del liberalismo político han propuesto diferentes modelos de razón pública para enfrentar la situación de desacuerdo moral permanente en las sociedades liberales. En este trabajo presentamos un modelo que defiende dos argumentos. Primero, argumentamos a favor de una interpretación deflacionista de las razones que son aceptables para los ciudadanos razonables. Segundo, introducimos una nueva terminología que distingue entre lo que llamamos razones dependientes, accesibles y aceptables. Sostenemos que sólo las segundas y las terceras son medios adecuados de discusión dentro de la razón pública, y las terceras son un estándar correcto de justificación de política pública.

PALABRAS CLAVE: liberalismo político, democracia deliberativa, justificación pública, legitimidad, desacuerdo

SUMMARY: Several authors have proposed different models of public reason as a way of dealing with the permanent moral disagreement of liberal societies. In this work we present a model defending two things. First, we defend a deflationist interpretation of the reasons that are acceptable to reasonable citizens. Second, we introduce a new terminology which distinguishes what we call dependent, accessible, and acceptable reasons. We hold that only the second and third types are adequate means for discussion within public reason, and the third type is a correct standard of justification of public policy.

KEY WORDS: political liberalism, deliberative democracy, public justification, legitimacy, disagreement

\section{Introducción}

Las sociedades liberales contemporáneas observan diferentes grados de pluralismo de concepciones morales. Algunas de estas concepciones forman elaboradas explicaciones sobre el origen y la estructura del valor moral. Entre éstas podemos contar a las distintas religiones - el catolicismo, el protestantismo, el islam, el judaísmo- y a las distintas teorías filosóficas —el kantismo, el utilitarismo, el realismo moral, el constructivismo-. Paradigmáticamente, Rawls (2005) se refirió a éstas como "doctrinas comprehensivas". Otras concepciones morales, la mayoría de ellas, no son tan articuladas como las 
anteriores. Estas últimas pueden variar tanto en la profundidad de sus explicaciones como en la flexibilidad de sus directrices morales. De hecho, muchos ciudadanos no podrían señalar con precisión qué forma parte de la concepción que suscriben hasta que alguna política pública parece atacarla o favorecerla.

Esta pluralidad genera un problema para las instituciones: normalmente ciudadanos que profesan diferentes concepciones morales exhiben fuertes desacuerdos sobre qué políticas públicas están justificadas (e.g., sobre la legalización, la regulación o la prohibición del aborto, la eutanasia, las drogas, el matrimonio entre personas del mismo sexo, el discurso de odio, las campañas políticas negativas, o sobre la política tributaria correcta). Este problema es todavía más grave si se considera que el pluralismo de concepciones morales es una condición permanente de las sociedades liberales que sólo puede ser mitigado utilizando las capacidades coercitivas del Estado.

Considerando este problema, los autores que suscriben el así llamado liberalismo político han sostenido que la discusión pública en las sociedades democráticas tiene que darse de acuerdo con el estándar de la razón pública (RP). A grandes rasgos, RP es una especificación de la deliberación democrática según la cual tanto los ciudadanos como los oficiales públicos en los tres sectores del estado deben esgrimir sólo razones que se funden en los valores y principios políticos básicos de las democracias liberales, así como omitir aquellas razones cuya fuerza normativa dependa de la aceptación de una concepción moral en particular. ${ }^{l}$ Generalmente, esta forma de entender RP sostiene que se debe distinguir entre "razones comprehensivas" (dependientes de una doctrina comprehensiva) y "razones políticas" (aquellas que articulan valores políticos básicos), y que la discusión pública sólo debe darse considerando las últimas. Igualmente, como señalan Bohman y Richardson (2009), otra formulación común de este entendimiento de RP es que en el debate público sólo deben ofrecerse "razones aceptables a todos los ciudadanos razonables", en donde ser razonable al menos requiere aceptar que la sociedad debe entenderse como un sistema de cooperación equitativo entre personas consideradas libres e iguales.

Sin embargo, a pesar de los esfuerzos de los liberales políticos para lograr precisión analítica sobre qué hace que una razón sea

${ }^{1}$ Véanse Nagel 1987, pp. 229-237, Gutmann y Thompson 1996, p. 66, Larmore 1999, p. 602, Macedo 2000, p. 13, Rawls 2005, p. 137, Estlund 2008, p. 41, Cohen 2009, pp. 52-58 y Quong 2011, pp. 256-290, para diferentes articulaciones de esta idea general de RP. 
comprehensiva o política, aún existe cierta indeterminación a este respecto que se refleja en constantes malentendidos por parte de los críticos de RP. Asimismo, la idea originalmente rawlsiana de que las razones propias de RP son aquellas que todos los ciudadanos razonables comparten también ha causado controversia.

En este contexto, en este trabajo tenemos dos objetivos principales. Primero, defenderemos un entendimiento deflacionista de lo que significa que una razón sea compartida por los ciudadanos razonables:

Tesis deflacionista: decir que una razón es compartida, consensuada, accesible, aceptable, por y para los ciudadanos razonables es una estipulación que significa que dicha razón se funda en (o es compatible con) valores políticos de una democracia liberal. La fuerza normativa de dicha razón reside exclusivamente en el hecho de que es una articulación de tales valores. Sostener además que es compartida por los ciudadanos razonables es sólo una manera de hacer patente que dicha razón, como consideración normativa adecuada, es comprensible o está al alcance de cualquier ciudadano que delibere de la forma correcta.

Consideramos que la tesis deflacionista es una primera clarificación necesaria para que un proyecto de RP sea exitoso. Los liberales políticos han exhibido cierta ambigüedad a este respecto. En algunos casos, estos autores parecen defender que la fuerza normativa de las razones propias a $\mathrm{RP}$ reside precisamente en el hecho de que son consensuadas, compartidas o aceptadas por los ciudadanos razonables de una sociedad liberal $-\mathrm{y}$ no exclusivamente en el hecho de que son articulaciones de los valores políticos que evitan consideraciones comprehensivas-.

Segundo, en este trabajo queremos contribuir a la clarificación del estándar normativo que RP impone. A nuestro parecer, los liberales políticos no han logrado notar con suficiente precisión que RP tiene tanto un propósito negativo como un doble propósito positivo. El propósito negativo de RP consiste en establecer qué razones no deben ser usadas ni en la deliberación ni en la justificación de leyes y políticas públicas concretas. Con respecto al doble propósito positivo, debe notarse que hay una diferencia normativa relevante en la labor de RP como estándar de discusión y como estándar de decisión. Para dar cuenta de este segundo objetivo, introduciremos una notación analítica diferente a la que prevalece en la discusión actual — que, como hemos mencionado, normalmente distingue 
entre "razones comprehensivas" y "razones políticas", o entre "razones comprehensivas" y "razones compartidas por los ciudadanos razonables"- Diferenciaremos entre lo que llamamos razones dependientes, accesibles y aceptables. Permítannos formular esta idea a la manera de la siguiente tesis:

Tesis del triple estándar: RP especifica un triple esquema normativo. Primero, RP ofrece un estándar sobre qué razones deben excluirse de la discusión pública (razones dependientes). Segundo, RP ofrece un estándar que nos dice qué razones son adecuadas para conducir la discusión pública (razones accesibles). Tercero, RP ofrece un estándar para saber cuáles de esas razones son adecuadas para justificar políticas públicas y leyes concretas (razones aceptables).

De este modo, la tesis del triple estándar defiende que los requisitos de RP correctamente entendidos consisten en la exclusión de las razones dependientes, en el compromiso de presentar exclusivamente razones accesibles, y en la disposición a someter las razones accesibles a la prueba de la discusión pública para identificar aquellas que sean aceptables como estándar de justificación de la política pública. Particularmente, la tesis del triple estándar hace hincapié en que RP no sólo ofrece las herramientas para saber qué razones deben excluirse de la discusión pública - como los liberales políticos tienden a enfatizar-. Dicho énfasis obvia que en el debate cotidiano en las democracias liberales la mayoría de las argumentaciones incorrectas articulan valores políticos y no comprehensivos. Como menciona la tesis del triple estándar, RP también ofrece las herramientas para distinguir cuáles de las razones no comprehensivas propias a la discusión pública ofrecen las mejores justificaciones de políticas públicas concretas.

Consideramos que tanto la tesis deflacionista como la tesis del triple estándar son aclaraciones necesarias para lograr que un modelo de RP sea exitoso. Como parte de nuestra defensa de la tesis del triple estándar, a lo largo de este texto hablaremos de las razones accesibles y las razones aceptables para los ciudadanos razonables. Sin embargo, tales locuciones tienen que ser entendidas a la luz de la tesis deflacionista: la fuerza normativa de estos dos tipos de razones no depende en lo absoluto de que sean compartidas (accesibles o aceptables) para los ciudadanos razonables. Como veremos, tales palabras son notaciones analíticas para referir a contenidos justificatorios concretos independientes al consenso de los ciudadanos razonables. 
$\mathrm{Su}$ uso, como la tesis deflacionista menciona, sólo hace referencia a la importancia de que tales razones pueden ser vistas como consideraciones normativas adecuadas por cualquier ciudadano que delibere de la forma correcta.

El trabajo sigue la siguiente estructura. En la segunda sección defenderemos la tesis deflacionista frente a otras dos interpretaciones de la fuerza normativa de las razones compartidas por los ciudadanos razonables - que llamaremos, respectivamente, la tesis del consentimiento actual y la tesis del consentimiento normativo-. En la tercera sección desarrollaremos la tesis del triple estándar y mostraremos cómo ésta ayuda a evitar ciertos malentendidos recurrentes por parte de los críticos de RP. Finalmente, en la cuarta sección presentaremos algunas aclaraciones sobre el modelo de RP que defendemos.

\section{La tesis deflacionista}

Para los liberales políticos, el poder coercitivo con que el Estado respalda las políticas públicas que adopta debe ser justificado a todos los ciudadanos razonables (Quong 2011, p. 290). Pero, ¿qué requisitos tiene que aceptar una persona para ser considerada como razonable? En la literatura actual existen muchas propuestas al respecto que varían en el grado de exigencias para ser considerado ciudadano razonable. Para los propósitos de este trabajo, comenzaremos adoptando una versión de lo que podría llamarse razonabilidad mínima. Un ciudadano es considerado razonable si:

(1) acepta que una sociedad democrática es un sistema de cooperación equitativa entre personas libres e iguales;

(2) acepta los valores y principios políticos básicos del liberalismo democrático (igualdad, libertad, tolerancia, equidad, reciprocidad, etc.);

(3) sólo brinda razones comunes a todos los ciudadanos razonables en las deliberaciones públicas.

A pesar de las divergentes formulaciones sobre razonabilidad que existen entre los autores afines al liberalismo político, todas ellas comparten este coto mínimo. ${ }^{2}$ De hecho, es muy común encontrar en

${ }^{2}$ Véase Wenar 1995 para una defensa de la razonabilidad mínima. Por su parte, Rawls 2005, Freeman 2007 y Quong 2011 defienden lo que puede llamarse razonabilidad rawlsiana: además de la razonabilidad mínima, un ciudadano razonable acepta que el uso de la razón práctica humana en condiciones de libertad está moldeado 
la literatura sobre liberalismo político y RP variaciones sobre la idea presentada en (3); esto es, la idea de que las razones adecuadas a la deliberación pública son aquéllas compartidas, accesibles, aceptables, consensuadas, por y para los ciudadanos razonables. Paradigmáticamente, Rawls sostuvo que:

Nuestro ejercicio del poder político es apropiado sólo cuando creemos sinceramente que las razones que ofreceríamos para nuestras acciones políticas $[\ldots]$ son suficientes, y cuando creemos razonablemente que otros ciudadanos pueden aceptar de manera razonable tales razones. (1999, p. 161)

Más recientemente, Cohen sostiene:

De acuerdo con ese ideal [RP], la justificación política, al menos en relación con ciertas cuestiones fundamentales, debe proceder en un terreno de discusión que pueda ser compartido. En el caso de la razón pública de la democracia, el terreno compartido consta de valores que pueden ser razonablemente compartidos por personas que se ven a sí mismas como libres e iguales, a pesar de sus desacuerdos doctrinales irreconciliables y sus desacuerdos sobre justicia $(2009$, p. 5, las cursivas son nuestras). ${ }^{3}$

Éstos son tan sólo dos ejemplos paradigmáticos que muestran cómo las razones propias a RP suelen identificarse como aquéllas consensuadas, compartidas, aceptables para ciudadanos razonables. Sin

por las cargas del juicio, y que tal ejercicio es a la vez el origen y la justificación del pluralismo de concepciones morales. Leland y van Wietmarschen (2012) sostienen lo que puede llamarse razonabilidad intelectual: además de la razonabilidad mínima, un ciudadano razonable debe aceptar que incluso agentes expertos situados en circunstancias ideales de reflexión pueden defender dos concepciones morales opuestas. Finalmente, Barry (1995) sostiene lo que podría llamarse razonabilidad escéptica: además de la razonabilidad mínima, un ciudadano razonable debe aceptar que no estamos en posición de conocer qué doctrina moral es correcta. Cada una de estas concepciones de razonabilidad añade exigencias normativas para ser considerado ciudadano razonable. A pesar de que no podremos extendernos al respecto en este texto, quisiéramos mencionar un problema de todas las concepciones de razonabilidad que van más allá de la mínima. A saber, que parecen implicar que un ciudadano razonable acepta en cierto grado la falibilidad de su propia concepción moral. Esto no sólo nos parece un requisito demasiado exigente para estar dentro del dominio de justificación, sino también que es un requisito incompatible con el liberalismo como tal, pues la libertad plena de conciencia implica que el estado no puede pedir a los ciudadanos que duden de la verdad de su propia doctrina.

${ }^{3}$ Todas las traducciones de los textos en inglés que no tienen una versión en castellano son nuestras. 
embargo, pasajes como los anteriores pueden generar la impresión de que la fuerza normativa de dichas razones se halla, precisamente, en el hecho que éstas son compartidas o consensuadas por las personas en su calidad de ciudadanos razonables. En la literatura se pueden identificar dos variaciones de esta idea. Permítannos enunciarlas como las siguientes tesis:

Tesis del consentimiento actual: las razones adecuadas a RP son aquéllas consentidas por una circunscripción actual de ciudadanos en las sociedades liberales contemporáneas; el consentimiento de dicha circunscripción les ofrece poder justificatorio.

Tesis del consentimiento normativo: las razones adecuadas a RP son aquéllas consensuadas entre una circunscripción idealizada y moralizada de los ciudadanos de una sociedad liberal consiente; el consentimiento de dicha circunscripción les ofrece poder justificatorio.

Estas dos tesis ofrecen una variación empírica y una normativa de la misma idea. Ambas proceden en dos etapas: primero, especifican el conjunto de ciudadanos cuyas razones deben ser consideradas para RP; segundo, estipulan que el consentimiento de esta circunscripción de ciudadanos ofrece la fuerza normativa a dichas razones.

Consideramos que ambas tesis están equivocadas. Comencemos con la tesis del consentimiento actual la cual interpreta los requisitos de razonabilidad como una cuestión que se debe determinar de manera empírica, como aquellas razones que de hecho los ciudadanos de las sociedades liberales contemporáneas comparten. Klosko ofrece una defensa de esta tesis:

Si el objetivo es descubrir principios que las personas puedan aceptar, entonces gran parte de ello depende de cuál sea su moralidad y sus visiones políticas, con las cuales los principios liberales deben encajar. Entonces tenemos que indagar qué es lo que creen los ciudadanos liberales. Para bien o para mal, la mejor evidencia disponible viene frecuentemente de la investigación mediante encuestas, sondeos de opinión pública, etc. (2000, p. 9)

En este pasaje, Klosko hace patente su aceptación de la tesis del consentimiento actual. Sin embargo, dicha tesis tiene un problema fundamental: a saber, hace que la fuerza normativa de las razones propias a RP dependa de las creencias contingentes de cierta circunscripción de ciudadanos actuales. Pero si es el consenso actual 
en tales ideas lo que las hace adecuadas para guiar la deliberación pública, su contenido parece volverse irrelevante. Si no existiera un consenso, por ejemplo, en que la libertad de expresión es algo que el Estado debe respetar y promover, entonces apelar a dicha libertad no sería una consideración válida en las discusiones públicas. Del mismo modo, si una religión fuera lo suficientemente consensuada en una sociedad liberal determinada, entonces — según la tesis del consentimiento actual - parecería seguirse que consideraciones dependientes de su aceptación podrían usarse en las deliberaciones públicas.

En términos generales, Klosko no repara en si la caracterización de ciudadano razonable adecuada a cualquier modelo de RP es normativa y no descriptiva. En este sentido, un ciudadano razonable es una idealización de un ciudadano común, un ciudadano que se conduce según (1), (2) y (3). Sin embargo, como se ha visto, aun aceptando que un ciudadano razonable es una caracterización normativa que especifica cómo deben comportarse los ciudadanos comunes en las democracias contemporáneas, se puede sostener que la fuerza normativa de las razones propias de RP surge del hecho de que son consentidas por esta caracterización idealizada de ciudadano. Esto es precisamente lo que la tesis del consentimiento normativo plantea. Quong ofrece una defensa de esta posición:

La legitimidad de los principios políticos no depende de si acaso los ciudadanos liberales actuales los aceptan, o de si tales principios son congruentes con sus creencias actuales. En cambio, los principios se definen como legítimos si es posible presentarlos de manera que los ciudadanos racionales y razonables puedan respaldarlos. (2011, p. 144)

En este pasaje Quong rechaza la tesis del consentimiento actual $\mathrm{y}$ defiende la tesis del consentimiento normativo. Como se ha visto, concordamos con Quong en que la primera tesis tiene que rechazarse. La fuerza normativa de las razones propias de RP no surge del consenso real de algún grupo de ciudadanos en las sociedades liberales. Sin embargo, creemos que Quong se equivoca al defender la segunda tesis. Según éste, la legitimidad de los principios políticos propios del liberalismo se debe a que dichos principios son aceptables para los ciudadanos razonables caracterizados de manera normativa. Como la anterior, esta posición se presenta en dos etapas. Primero, especifica al conjunto de ciudadanos razonables: en este caso, se ofrece una caracterización normativa de ellos. Segundo, estipula que el consentimiento de esta versión idealizada de los ciudadanos ofrece 
la legitimidad de los principios políticos. Sin embargo, esta posición tiene el siguiente problema de fondo: ¿cuál es el poder justificatorio de tal estipulación? ¿Por qué la estipulación del consenso de una entidad idealizada tiene fuerza normativa — en el caso de Quong, la facultad de ofrecer legitimidad a ciertos principios; en el caso que nos ocupa más directamente, la facultad de validar razones para justificar políticas públicas concretas-?

Estas preguntas originan un dilema para un defensor de la tesis del consentimiento normativo. Vamos a presentarlo a través del siguiente caso. Supongamos que dos actores políticos, $A$ y $B$ (que se rigen por el estándar que impone RP — ciudadanos, oficiales políticos o jueces en deliberaciones públicas-) sostienen posiciones encontradas sobre si una política pública concreta, $x$, está justificada. $A$ defiende que $x$ está justificada y $B$ defiende lo opuesto. Supongamos además que $A$ está en lo correcto y, según el estándar impuesto por las razones de RP propias para este caso, $x$ está justificada. ¿Cómo puede el defensor de la tesis del consentimiento normativo explicar a $\mathrm{B}$ que $x$ está justificada? Dicho defensor tiene dos opciones: o argumenta que $x$ está justificada meramente debido a que sería aceptada por una circunscripción idealizada de ciudadanos (los ciudadanos razonables), o brinda razones para mostrar que $x$ articula de mejor forma los valores políticos básicos del liberalismo en su conjunto y debido a ello sería aceptada por los ciudadanos razonables. Si elige la primera opción, entonces este defensor tiene que aceptar que su tesis ofrece una explicación a $B$ que cae en petición de principio, pues $B$ podría simplemente sostener lo opuesto. Si elige la segunda opción, entonces tiene que aceptar que su tesis es trivial para explicar a $B$ porqué $x$ está justificada, pues tal explicación recae exclusivamente en las razones ofrecidas para mostrar que $x$ articula de la mejor forma los valores políticos básicos del liberalismo.

El defensor de la tesis del consentimiento normativo podría tratar de responder al dilema mencionado sosteniendo que $x$ está justificada debido a que es la mejor articulación de los valores políticos y también debido a que sería aceptada por los ciudadanos razonables, como si esto último añadiera una nueva razón para sostener que $x$ está justificada. Sin embargo, al ofrecer esta respuesta, este defensor se compromete a mostrar qué añade a la justificación de $x$ el consenso hipotético de los ciudadanos razonables en torno a ella. Esto debido a que dicho consenso es estipulado una vez que se muestra que las razones que articulan valores políticos favorecen a $x$. De modo que volvemos a la pregunta fundamental con la que empezamos: ¿cuál es la fuerza normativa o poder justificatorio de tal estipulación? 
Consideramos que el dilema anterior es una objeción insalvable contra la tesis del consentimiento normativo. Apelar al consenso de ciudadanos idealizados es a la vez equivocado e innecesario para fundamentar el poder justificatorio de las razones propias de RP. Desde un punto de vista sustantivo, basta con señalar que dicho poder reside exclusivamente en los valores políticos liberales y las consideraciones consistentes con ellos. En concreto, pues, apelar al consenso idealizado es un mecanismo de justificación insatisfactorio porque expresa ya sea una petición de principio o una trivialidad.

Bohman y Richardson (2009, pp. 263-264) han desarrollado algo parecido a esta crítica. Sin embargo, resulta extraño que estos autores terminen defendiendo una variante de la tesis del consentimiento normativo según la cuál las razones propias de RP tienen poder justificatorio debido a la "esperanza razonable de lo que ciudadanos razonables de hecho [las] aceptarán" (2009, p. 267, las cursivas son nuestras) como justificaciones de políticas públicas. Según estos autores, que montan una crítica feroz a la idea de que la fuerza justificatoria de las razones propias de RP provenga de que los ciudadanos razonables, ya sea reales o idealizados, puedan aceptarlas, terminan defendiendo una simple variación según la cual dichos ciudadanos en su versión idealizada de hecho aceptan tales razones - y esto les confiere poder justificatorio-. Cómo definen estos autores la circunscripción de los ciudadanos razonables (ya sea de manera empírica o idealizada) no es muy claro y discutirlo no es necesario para los propósitos de este artículo. Esto se debe a que Bohman y Richardson no se dan cuenta de que el problema con estas interpretaciones de las razones adecuadas a RP no es la modalidad del consenso de los ciudadanos razonables, sino la apelación al consenso en sí mismo - tal cual lo muestra el dilema mencionado-.

De este modo consideramos que la mejor interpretación de (3) es la tesis deflacionista - que presentamos en la introducción-. La fuerza normativa de las razones propias de RP recae exclusivamente en el hecho de que dichas razones articulan valores políticos básicos o consideraciones compatibles con ellos. No añade nada a la justificación de las políticas basadas en tales razones de que cierta circunscripción de ciudadanos reales las acepten, o que estipulemos que cierta circunscripción idealizada de ciudadanos las aceptan o aceptarían - como la tesis del consentimiento actual y las variaciones de la tesis del consentimiento normativo plantean, respectivamente-. Desde el punto de vista de la justificación, los valores políticos y las consideraciones compatibles con ellos son suficientes. Esta forma de entender (3), por otro lado, ofrece una respuesta directa a las críticas que Enoch (2013, 
pp. 164-170) vertió recientemente. Él enfoca su crítica en todas las posiciones de la filosofía moral (y no sólo sobre RP) que apelan al consentimiento de una versión idealizada del agente. En tanto que dicho consentimiento no desempeña un papel justificatorio según la tesis deflacionista que defendemos, la crítica de Enoch a RP no se aplica a nuestro modelo.

Ante esto surge la pregunta: ¿por qué continuar sosteniendo que las razones propias de RP son aquéllas consensuadas, accesibles, aceptables, compartidas, por y para los ciudadanos razonables y no simplemente decir que son las que articulan valores políticos liberales? Como veremos en la siguiente sección, nuestro modelo de RP refiere a las razones adecuadas para la deliberación pública como accesibles y aceptables para los ciudadanos razonables. A pesar del nulo poder justificatorio de esta idea, sostener que las razones propias de RP son accesibles y aceptables para esta circunscripción idealizada de ciudadanos cumple otra función: a saber, que las razones adecuadas para RP, y las justificaciones concretas que se basen en ellas, deben poder comprenderse y verse como consideraciones normativas válidas por cualquier ciudadano que razone de manera correcta. De ahí que tanto la tesis deflacionista como la tesis del triple estándar hagan hincapié en mantener tal nomenclatura, en lugar de eliminarla por completo.

\section{La tesis del triple estándar}

Hemos visto que la gran mayoría de los autores afines al liberalismo político sostiene un modelo de RP según el cual las razones adecuadas para la deliberación pública son aquellas que los ciudadanos razonables comparten. ${ }^{4}$ Ante ello, en la sección anterior defendimos una forma deflacionista de entender esta idea - en la que el consenso de dicha circunscripción no desempeña un papel en la justificación-. En esta sección queremos contribuir a clarificar de manera analítica los propósitos propios de RP, ya que éstos nos parecen subdesarrollados en la literatura contemporánea.

Como ya hemos mencionado, introduciremos una distinción entre lo que denominamos razones dependientes, razones accesibles y razones aceptables. Consideramos que esta distinción es fundamental para especificar tres cuestiones. Primero, cuáles son las razones que, según RP, deben permanecer fuera de la discusión pública. Segundo,

${ }^{4}$ Véase Nagel 1987, pp. 229-237, Larmore 1999, p. 602, Macedo 2000, p. 13, Rawls 2005, p. 137, Cohen 2009, pp. 52-58, Quong 2011, pp. 256-290. 
por qué debe respetarse la limitación normativa de ofrecer razones accesibles a todos los ciudadanos razonables en la deliberación pública. Y, tercero, qué razones deben servir como estándar de justificación de la política pública.

\section{Razones dependientes}

En nuestro modelo, las razones dependientes siempre deben ser excluidas de la deliberación pública. Esta idea se corresponde con el propósito negativo de RP (i.e., especificar qué razones no se deben usar en la deliberación pública). Sin embargo, a pesar de ser inadecuadas como estándar público de discusión y justificación en una sociedad liberal, dichas razones tienden a proliferar en la sociedad debido a su vínculo con prácticas y tradiciones específicas que están presentes en su interior. Una razón es dependiente si articula:

(D1) consideraciones comprehensivas, o

(D2) consideraciones empíricamente falsas, altamente improbables o indemostrables, o

(D3) consideraciones fundadas en prejuicios sociales extendidos.

Ésta es una caracterización disyuntiva de lo que es una razón dependiente. Ocupémonos de (D1). Una consideración comprehensiva es aquélla cuya fuerza normativa depende de la aceptación de lo que Rawls llama una doctrina comprehensiva particular (2005, p. 38). Como mencionamos en la introducción, los ejemplos más paradigmáticos de doctrinas comprehensivas son las religiones (el catolicismo, el protestantismo, el islam, el judaísmo) y las filosofías morales (el kantismo, el utilitarismo, el realismo moral, el constructivismo). El modelo de razón pública que Rawls defiende hace hincapié en cómo dichas consideraciones, debido al hecho del pluralismo razonable, no pueden fungir como un estándar de justificación de política pública en una democracia liberal. Así, por ejemplo, al discutir sobre la posible legalización, prohibición o regulación del aborto, la consideración de que el alma humana entra al cuerpo desde la concepción (Rogel 1977) es una razón comprehensiva, pues su fuerza normativa depende de la aceptación de una doctrina comprehensiva particular que se compromete con la existencia del alma y la forma en que ésta entra al cuerpo humano.

Sin embargo, existen consideraciones que son inadecuadas para la discusión pública y no forman parte de doctrinas comprehensivas 
particulares. Éstas pueden ser, como (D2) especifica, razones cuyas credenciales empíricas son dudosas. Por ejemplo, en Francia en el debate sobre la posible legalización de la adopción infantil por parte de parejas homoparentales, una de las consideraciones vertidas fue la de que un niño criado por dos padres del mismo sexo no generaría los complejos de Edipo (si es hombre) o de Electra (si es mujer), pues para su formación necesita crecer bajo la tutela de una pareja heteroparental. Dada la importancia de tales complejos en la formación de la psique humana, de acuerdo con este razonamiento, permitir la adopción a parejas homoparentales no es recomendable (Robcis 2013). Esta razón articula consideraciones de corte sicoanalítico que, si bien no forman parte de una doctrina comprehensiva en particular, son igualmente inadecuadas como estándar de justificación de política pública, pues son difíciles de comprobar empíricamente (Eysenck 2004). Del mismo modo, otro ejemplo de razones dependientes no comprehensivas la ofrece la argumentación de George a favor de prohibir la prostitución. Según este autor, cuando una persona participa de la prostitución daña "las bases más sólidas para ser maridos y padres de familia", lo que a la larga repercute en la estabilidad del matrimonio como institución social — afectando la propia subsistencia de la sociedad - Este razonamiento es problemático por dos razones: primero, que la prostitución tenga el efecto en el matrimonio que George describe es una cuestión empírica controvertida. Aun cuando pueda ser verdad que ciertos matrimonios se vean afectados por que el padre de familia sea cliente asiduo de la prostitución, no hay evidencia concluyente de que esto conduzca a que exista una afectación amplia y significativa en la institución del matrimonio en general. Segundo, a pesar de que el matrimonio puede ser útil para los fines de la reproducción social, es una cuestión empírica debatible si dicha institución es necesaria para tales fines. Otro ejemplo más de razones dependientes no comprehensivas es el que ofrecen los ciudadanos que defienden el movimiento antivacunas, y que afirman que las vacunas múltiples más exitosas y aquellas contra la polio o el sarampión pueden causar autismo. Una vasta evidencia empírica sugiere que esta idea es simplemente incorrecta (DeStefano 2006). Ejemplos como los anteriores revelan que no necesariamente todas las razones inapropiadas para $\mathrm{RP}$ son de corte comprehensivo. A pesar de ello, estas consideraciones son medios inadecuados para los fines públicos del Estado debido a su falsedad o a su carácter de indemostrabilidad empírica.

El último tipo de razón dependiente, (D3), refleja la existencia de prejuicios sociales, los cuales pueden más o menos estar generalizados 
al interior de un grupo social o de la sociedad en su conjunto. Con la finalidad de aclarar en qué consiste un prejuicio social, hay que decir que su presencia pública tiene sustento al hacer algún tipo de diferenciación o exclusión (de estatus político o social, de derechos o de libertades básicas) cuando no existe una buena razón para hacerlo. La preservación de dichos prejuicios sociales puede deberse a continuar con una creencia o una práctica de forma irreflexiva. Esto genera estereotipos de papeles sociales que pueden ser muy dañinos para la igualdad ciudadana. Por ejemplo, la idea de que los funcionarios públicos, especialmente aquellos que tienen que dar la cara por las instituciones deban tener ciertas características físicas excluyendo así a quienes tienen tatuajes visibles. ${ }^{5}$ Esto no significa que las personas con tatuajes sean menos competentes en dichos puestos, sino simplemente dan "mala imagen".

A pesar de que este razonamiento no forma parte directa de una doctrina comprehensiva ni plantea una tesis empíricamente problemática (al menos no en el mismo sentido que la consideración sicoanalítica ofrecida en el debate francés sobre la crianza homoparental o las consideraciones ofrecidas por el movimiento antivacunas), se le tiene que excluir de la discusión pública debido a que las expectativas sociales concernientes a la imagen y al "buen gusto" no pueden ser un estándar de deliberación ni de justificación de política pública. Muchos de los estereotipos sociales generan razones dependientes de este tipo, todas ellas inadecuadas para justificar políticas públicas.

Analizar estos tres tipos de razones dependientes nos ofrece, por ejemplo, una consideración fuerte en contra del modelo de RP promovido por Gaus (1996). De acuerdo con este modelo, una política pública es correcta cuando diferentes razones comprehensivas convergen en su justificación. Así, por ejemplo, distribuir de manera equitativa la libertad de expresión entre mujeres y hombres, según el modelo de Gaus, puede encontrar varias justificaciones (Dl) convergentes. Sin embargo, debe tenerse en cuenta que las diferentes concepciones morales en la sociedad no sólo ofrecen razones de tipo (D1), sino también de tipo (D2) y (D3). ¿Acaso sería conveniente que las diferentes concepciones morales utilizaran razones (D3) convergentes para justificar la distribución equitativa de la libertad de expresión entre los géneros? Una de ellas, por ejemplo, podría defender la libertad de expresión de las mujeres con la argumentación de que escuchar hablar a las mujeres aumenta su sex-appeal frente al

${ }^{5}$ A manera de ejemplo, véase el Reglamento Interno de la Secretaría de Seguridad Pública del Estado de Guadalajara: http://portal.guadalajara.gob.mx. 
deseo masculino. Ésta es una razón (D3) que, aunque pueda utilizarse a favor de la distribución equitativa de la libertad de expresión entre los géneros, es contraria al valor político de promover la igualdad en el estatus de ciudadanía para todas las personas, pues tiene como consecuencia indeseable subordinar los intereses de un grupo de ciudadanos (las mujeres) a los intereses de otro (los hombres). De este modo, además de enfrentar las objeciones comunes presentes en la literatura (Quong 2011, pp. 265-281), el modelo de convergencia de Gaus tiene que explicar por qué las diferentes leyes y políticas públicas no deben buscar la convergencia de razones (D3) — que también forman parte del imaginario de las diferentes concepciones morales dentro de las sociedades liberales-.

\section{Razones accesibles}

Según nuestra propuesta la relación entre no dar razones dependientes y dar razones accesibles es estrecha aunque evitar las primeras no agota, por sí mismo, todos los requisitos que las segundas implican. En esto consiste el primer propósito positivo de RP: en ofrecer un estándar de deliberación que especifique las razones correctas para conducirla. Queremos enfatizar que la referencia a la "accesibilidad" de las razones es técnica y no está relacionada con el uso cotidiano que este término puede adquirir en un momento dado. Esto nos distancia de la tesis del consentimiento actual: el requisito de dar razones accesibles a los ciudadanos razonables no equivale a ofrecer cualquier razón que pueda ser entendida y consensuada directamente por el grueso de la ciudadanía de una sociedad liberal determinada. Por el contrario, tal como defendimos en la primera sección, la accesibilidad de las razones propias de RP es esencialmente normativa. Éstas son consideraciones que constituyen interpretaciones válidas de los valores políticos que están en juego en la deliberación pública. A pesar de esta aclaración conceptual, como hemos mencionado, creemos que señalar estas razones como accesibles conserva el sentido de "acceso fácil" o "inteligibilidad" que las justificaciones públicas deben tener. En concreto, una razón es accesible si:

(Al) No es dependiente.

(A2) Emplea consideraciones que en principio pueden hacerse inteligibles ante cualquier ciudadano (que cuente con mínimas capacidades morales y de juicio).

(A3) Se basa en (o no se opone a) conclusiones científicas no controvertidas. 
(A4) Articula (o no se opone a) valores políticos básicos de una democracia liberal.

Sostenemos que los requisitos (A1), (A2), (A3) y (A4) son individualmente necesarios y conjuntamente suficientes para que una razón se considere accesible.

El primer requisito para que una razón sea accesible —i.e., (A1) es que ésta no sea dependiente. Esto es, una razón accesible no puede emplear consideraciones comprehensivas, de carácter empíricamente cuestionable, o fundarse en prejuicios sociales ampliamente extendidos. Como se ha visto, tales razones no pueden ser un estándar adecuado de justificación pública en una democracia liberal.

Por otro lado, (A2) aclara algo importante. Varios autores han criticado la idea de razón pública argumentando que muchas razones que son justificaciones adecuadas de política pública son altamente complejas debido a su carácter técnico. ${ }^{6}$ Dicho carácter logra que sólo expertos con una preparación sofisticada puedan comprenderlas correctamente. Considérese un ejemplo cuando se empezó a discutir si la política pública sobre salud sexual debía o no fincarse en la promoción del uso del condón; sólo los científicos que colaboraron en su diseño estaban en condiciones de entender, de primera mano, las razones técnicas que sustentan que el uso del condón es confiable para conseguir los fines señalados (Berer 2006). Por su carácter altamente técnico, sería un grave error excluir dichas razones de la discusión pública. Sin embargo, creemos que para que una razón sea un estándar adecuado de deliberación y de justificación pública, al menos en principio, debe ser inteligible para cualquier ciudadano razonable que tenga las capacidades morales y de juicio mínimas, pues todos los ciudadanos razonables sufrirán las consecuencias de la política pública en cuestión.

Por su parte, en relación con (A3) es importante precisar que el tipo de ciencia a la que nos referimos es, en gran medida, la ciencia experimental (ya sea la ciencia natural o la ciencia social) o aquélla cuyas predicciones son confiables por la utilización de correlaciones estadísticas o muestras representativas adecuadas. Las razones (A3) deben fundarse en el conjunto de estándares que los practicantes de la ciencia comparten y cuya función es establecer la probabilidad de que ocurra un fenómeno. Como Greenawalt menciona: "si la investigación científica puede establecer con certeza la probabilidad de algo,

${ }^{6}$ Véanse George 2000, p. 52, Waldron 1993, p. 839, Greenawalt 1988, p. 49, Gaus 1996, pp. 132-136. 
entonces ese algo se establece de acuerdo con las razones públicamente accesibles" (1988, p. 58). El éxito de la capacidad predictiva de dichos estándares da como resultado que su confiabilidad no esté sujeta a duda razonable.

Otra cuestión que cabe resaltar es que las razones (A3) tienen que ser claramente diferenciadas de las razones seculares. A pesar de que Rawls (1999, p. 167) distingue - aunque apresuradamente — las razones propias de $\mathrm{RP}$ de las razones directamente seculares, la falta de precisión en torno al significado del requisito de accesibilidad da la impresión, bastante generalizada, de que uno de los principales propósitos de RP es preservar la racionalidad secular a través de la exclusión de cualquier fundamento religioso (Vallier 2011, p. 367). Varios autores han señalado que dicho propósito es altamente problemático no sólo porque favorece arbitrariamente a la racionalidad secular, sino porque niega la importante contribución histórica del pensamiento no secular en la instauración de los valores del liberalismo. Carter (1987, p. 990), por ejemplo, pregunta si todas las conclusiones de las ciencias, en contraste con las de la "conciencia", son adecuadas para entender problemas de injusticia. A partir de los ejemplos de injusticias que la racionalidad secular puede cometer (como los intentos seudocientíficos de mostrar la inferioridad natural de una minoría étnica o de la mujer (Clarke 1873)), concluye que el liberalismo ha beneficiado discrecionalmente a la ciencia por encima de otro tipo de racionalidades - como el creacionismo - que también podrían ayudar a que la sociedad preserve términos de cooperación justos. $^{7}$ Sin embargo, es un error pensar que las razones accesibles sean las seculares en detrimento de las religiosas sólo en virtud de que son más afines al tipo de lenguaje que se utiliza en las ciencias. Las razones seculares, al igual que las religiosas, pueden ser incorrectas o simplemente inapropiadas de ser consideradas en el debate público si no se les somete al examen del estándar de deliberación y evidencia que aquí defendemos. Por lo tanto, dichas razones no pueden verse como un dogma.

Finalmente, el requisito (A4) especifica las razones con contenidos liberales adecuadas a la deliberación. En última instancia, el uso de estas razones se justifica (como el propio sistema político liberal) en la importancia de tratar a todos los ciudadanos como libres e iguales. Éstas son articulaciones del valor de la libertad, la igualdad de oportunidades, la tolerancia, la reciprocidad, la eficacia, la estabilidad, etc.

${ }^{7}$ Para una discusión del mismo punto véase Waldron 1993, pp. 842-848. 


\section{Razones aceptables}

En una democracia liberal, la deliberación sólo debe estar orientada por razones accesibles. Éstas conforman los materiales adecuados para el intercambio público de opiniones y posturas. Sin embargo, RP tiene un segundo propósito positivo: además de establecer un estándar de deliberación (limitarse a ofrecer razones accesibles), RP establece un estándar de justificación de política pública. Este segundo estándar constituye una exigencia normativa superior. Para que una política pública esté justificada, dicha justificación no sólo debe fundarse en razones accesibles; éstas tienen que configurar la mejor articulación de los valores políticos según sea el caso. Es necesario, pues, evaluar cuál de las distintas opciones de ponderación pública que se fundan en razones accesibles es la más razonable para ser adoptada. Es así que una razón es aceptable si:

(ACE1) Es una razón accesible.

(ACE2) Es una ponderación suficientemente plausible de los valores políticos de una democracia liberal (y de las consideraciones compatibles con éstos) para contar como estándar público de justificación.

Como establece (ACE1), la primera característica de una razón aceptable es que sea accesible. Partiendo de esto, la función de la deliberación pública tiene que entenderse como una manera de poner a prueba las diferentes argumentaciones basadas en razones accesibles, con el fin de establecer cuál de ellas es la mejor. Algunas tendrán que ser desechadas, no sólo porque no incorporan alguno de los valores políticos centrales, sino porque no representan un balance de ponderación lo suficientemente bueno o competitivo frente a otras opciones de ponderación. Rawls (1999, p. 194) señala algo como esto de manera apresurada: una doctrina comprehensiva particular, incluso si ofrece razones basadas exclusivamente en los valores políticos a favor de determinada política pública, puede no brindar la mejor articulación de tales valores. En nuestra terminología, dicha doctrina comprehensiva está cumpliendo con el estándar deliberativo de RP al ofrecer razones accesibles; sin embargo, después de haberlas sometido a la prueba del debate y de la discusión pública junto con el resto de las opciones disponibles en el caso, dichas razones no cumplen con el estándar de justificación propio de RP, de modo que no son aceptables. 
4. Hacia un nuevo modelo de razón pública

En el siguiente esquema se puede visualizar el modelo de razón pública que proponemos:

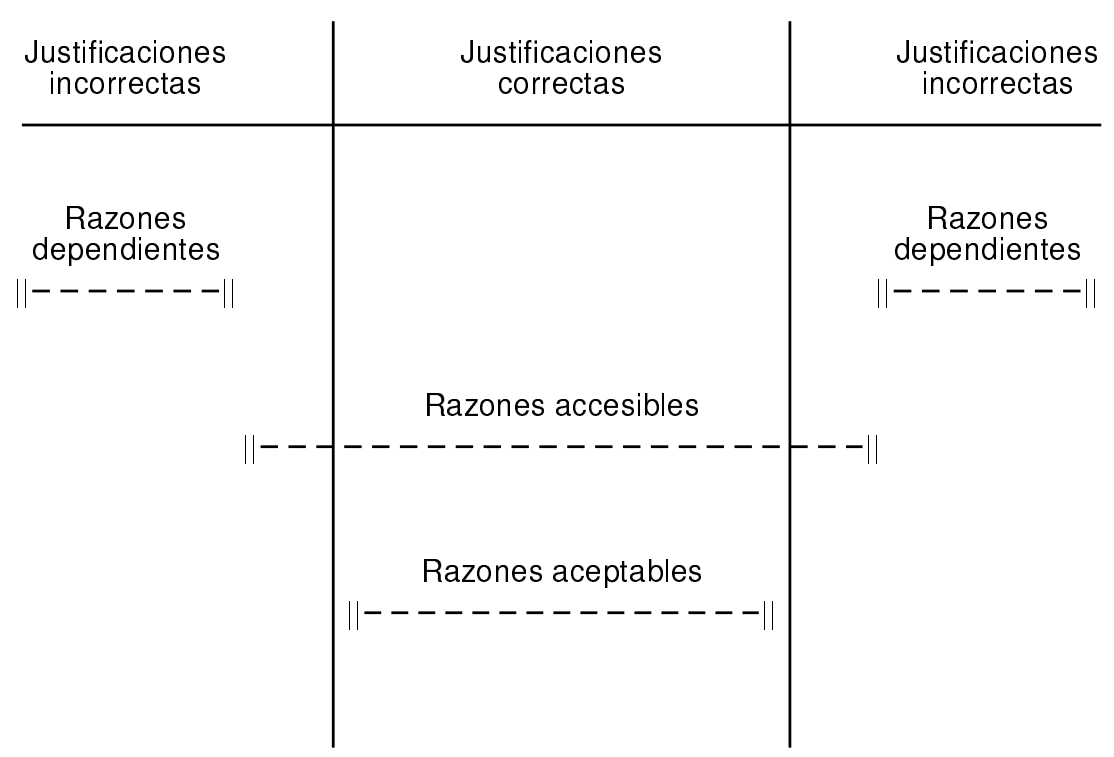

Esquema 1

En esta última sección queremos resaltar cinco clarificaciones del modelo de RP que defendemos - aunque, por falta de espacio, no podremos desarrollarlas a profundidad aquí-.

La primera clarificación, como se ve en el esquema 1, muestra que existen dos maneras de identificar la existencia de justificaciones incorrectas. La primera es cuando los ciudadanos o representantes públicos no ofrecen razones accesibles, ya sea porque presentan contenidos comprehensivos a manera de fundamento de sus posturas o porque ofrecen conclusiones científicas muy controvertidas. Es decir, este tipo de incorrección se deriva del hecho de que los ciudadanos presentan razones dependientes - (D1), (D2) o (D3) - y no accesibles -(A2), (A3) o (A4)—, de la manera en la que las hemos caracterizado.

El segundo tipo de justificación incorrecta que aquí proponemos supone que los ciudadanos o representantes públicos de una sociedad no puedan presentar a manera de postura pública defendible una 
ponderación o balance que incorpore, de forma lo suficientemente satisfactoria, los valores políticos centrales. La literatura sobre el liberalismo político tiende a hacer énfasis sólo en el estándar de ofrecer razones accesibles. Sin embargo, evitar la segunda forma de incorrección que aquí señalamos es tan importante como evitar la primera. Muchas argumentaciones inadecuadas articulan exclusivamente valores políticos en infinidad de debates. Cuando los estados liberales discuten cómo encarar la violencia política pasada reciente, suele argüirse que el valor de la estabilidad justifica la falta de indagación y rectificación histórica - especialmente si los perpetradores siguen vivos (Elster 2004) - . Las posiciones radicales defienden que el aborto se justifica en cualquier momento antes del nacimiento del neonato debido a que la libertad de la mujer para decidir sobre su cuerpo tiene prioridad absoluta, no sólo ante una célula cigótica o un embrión menor a los tres meses, sino también ante un feto sano incluso con ocho meses de gestación (Tooley 1984). Los libertarios defienden la desregularización absoluta del mercado financiero y apelan al derecho al intercambio de la propiedad privada (Friedman 2002). Varios gobiernos democráticos cometen repetidamente serias violaciones a la privacidad ciudadana apelando a la segurida. ${ }^{8}$ Todas estas políticas públicas (amnistías a perpetradores de violencia política pasada, la despenalización del aborto hasta el noveno mes de gestación, la desregularización total del mercado financiero, la vigilancia estatal sobre la vida privada de los ciudadanos) pueden justificarse exclusivamente en valores políticos básicos afines a las democracias liberales. A pesar de esto, parece claro que las argumentaciones revisadas son incorrectas. Lo anterior se debe, en todos los casos, a que tales argumentaciones no cumplen con el requisito (ACE2). Cada una de ellas falla al tratar de ofrecer una ponderación adecuada del conjunto de valores políticos - ya que se concentra en priorizar sólo uno de ello-. Que estas argumentaciones defectuosas sean tan comunes en las sociedades democráticas destaca la importancia de mostrar cómo RP también ofrece un estándar de justificación de acuerdo con (ACE2) que las descalifica. De hecho, en el debate cotidiano en las democracias liberales, se constata reiteradamente que la mayoría de las argumentaciones incorrectas articulan valores políticos y no comprehensivos. Las discusiones más comunes se centran en la interpretación de las implicaciones concretas de los valores políticos para ciertos temas. Esta realidad cotidiana muestra que la literatura sobre RP y el liberalismo político

\footnotetext{
${ }^{8}$ Véase http://www.nytimes.com/roomfordebate/2013/06/09/is-the-nsa-surveillance-threat-real-or-imagined
} 
se ha equivocado al hacer énfasis exclusivamente en el desarrollo de porqué las argumentaciones comprehensivas son incorrectas.

La segunda clarificación, de acuerdo con nuestro modelo de RP, señala que en la gran mayoría de las discusiones sobre política pública no hay una sola respuesta correcta. En nuestra terminología, debe decirse que hay un pluralismo de razones aceptables $-\mathrm{o}$, si se prefiere, un pluralismo de argumentaciones aceptables-. Esto significa dos cosas a la vez.

Por un lado, a una misma política pública la justifican diferentes conjuntos de razones aceptables. D'Agostino (1996, p. 30) distingue entre lo que llama el modelo del "consenso fuerte" y el modelo del "consenso débil". Según el primero, todos los ciudadanos deben aceptar una política pública determinada por las mismas razones. Según el último, los ciudadanos pueden aceptar dicha política por diferentes razones. Nuestra interpretación de RP es un ejemplo del segundo modelo, siempre y cuando las razones en cuestión sean todas aceptables. Esto también toma en cuenta la importancia de los diferentes contextos de las sociedades liberales contemporáneas. Por ejemplo, sería un error pensar que la controversia del aborto debe resolverse exactamente de la misma manera y atendiendo exactamente las mismas consideraciones y valores políticos en países con tantos contrastes económicos, históricos y culturales como México y Estados Unidos. Así, pues, puede haber variación contextual de razones aceptables sobre una misma política pública.

Por otro lado, en infinidad de casos puede ser que más de una política pública concreta sea correcta. Las razones aceptables identifican un conjunto de políticas públicas que son lo suficientemente justas. Quizá en cada discusión haya una sola alternativa que ejemplifique la justicia perfecta. Sin embargo, el pluralismo de las sociedades liberales tiene como consecuencia permanente el desacuerdo en este aspecto. Piénsese en una deliberación sobre política tributaria. Una posición puede argumentar que, en función de la importancia de la redistribución del ingreso para mantener la igualdad material y de oportunidades de los ciudadanos, la tasa de impuesto a la clase social más favorecida debe ser del 50 por ciento. Otra posición, en cambio, ante la ponderación del valor de la igualdad ciudadana y el derecho de dicha clase sobre sus bienes, puede argumentar que la tasa adecuada es del 45 por ciento. A nuestro parecer, puede darse el caso de que ambas políticas cumplan con el estándar de aceptabilidad de RP. Otros casos comunes de pluralidad de políticas públicas correctas tienen que ver con el alcance de la libertad de 
expresión y la regulación de las campañas negativas. En estos casos, hay un rango de variación aceptable que no se puede determinar fuera de contexto (Brettschneider 2012). A pesar de que es claro que varias prácticas no pueden incluir dicha libertad en ninguna sociedad liberal (p.ej., el discurso de odio que incita a la violencia), el desarrollo institucional concreto, la historia de la sociedad democrática en cuestión, el entorno cultural y otras contingencias determinan en parte el rango de políticas sobre lo que sí puede estar incluido. En todos estos casos, lo importante es mostrar que la política pública en cuestión articula y pondera de forma adecuada los valores políticos básicos y, en esa medida, es suficientemente justa. Que la discusión pública sea conducida teniendo en cuenta la directriz de estos valores no quiere decir que existan recetas o listas completas que nos lleven a soluciones políticas unívocas e invariables en todas las sociedades liberales. Por ello, puede decirse que RP en última instancia es un estándar de suficiencia de justicia que acepta múltiples variaciones.

Entender a RP como un estándar de justificación compatible con una pluralidad de soluciones concretas suficientemente justas ayuda a evitar algunos malentendidos altamente extendidos. Paradigmáticamente, Sandel (1998) objeta que RP no puede eliminar los desacuerdos que existen en torno a las cuestiones de justicia - tanto como no se pueden resolver los desacuerdos sobre aquello en lo que consiste la vida buena-. Ante esta crítica tienen que señalarse dos cosas. En primer lugar, como Quong (2011, pp. 192-220) sostiene hay que distinguir los desacuerdos en torno a la justicia de los desacuerdos sobre la vida buena. Quong denomina al primer tipo "desacuerdo justificativo" y al segundo "desacuerdo fundacional". Un desacuerdo justificativo se da cuando "los participantes comparten ciertas premisas que sirven como un estándar mutuo y aceptable de justificación pero están en desacuerdo acerca de ciertas conclusiones sustantivas" (2011, p. 193). El desacuerdo fundacional se caracteriza por "el hecho de que los participantes no comparten ninguna premisa que pueda servir como un estándar mutuo y aceptable de justificación" (2011, p. 193). Así, los desacuerdos en torno a la vida buena y los que se presentan en torno a la justicia son cualitativamente distintos. En segundo lugar, también debe señalarse que no es la función de RP eliminar por completo el desacuerdo en la sociedad sobre las cuestiones sustantivas de justicia. Nuestro modelo hace hincapié en esto al sostener que puede haber más de una justificación correcta de una misma política pública, y también más de una política pública correcta sobre un asunto determinado. El desacuerdo sobre cuál es la mejor justificación, en el primer caso, o sobre cuál de las 
políticas suficientemente justas ejemplifica la justicia perfecta, en el segundo caso, persistirá. Sostener que la razón pública es completa (i.e., que los materiales que contiene -(A2), (A3) y (A4), en nuestra terminología - son suficientes para determinar en todos los casos qué política pública está justificada - Quinn 1995, Reidy 2000, Greenawalt 1988, Gaus 1996, Schwartzmann 2004, Williams 2000-) no implica comprometerse con la eliminación del desacuerdo. Por el contrario, el punto central es saber cuándo nuestras leyes y medidas públicas están justificadas inclusive frente al desacuerdo persistente que pueden mostrar algunos de los ciudadanos razonables sobre lo que la justicia perfecta requiere.

La tercera clarificación de nuestro modelo de RP tiene que ver con la relación entre la justicia, la legitimidad y la justificación. Muchos autores en la tradición liberal debaten sobre esta relación. Wenar (2004), por ejemplo, sostiene que en realidad la teoría rawlsiana tiene que entenderse como una teoría de la legitimidad y no como una teoría de la justicia. Varios autores afines al liberalismo político sostienen algo similar -i.e., que la preocupación central de RP recae en mostrar la legitimidad del poder coercitivo del estado-. Otros ponen el énfasis en la justificación (Simmons 2001). Según nuestro modelo, la relación entre estos tres conceptos se da de acuerdo con el siguiente esquema:

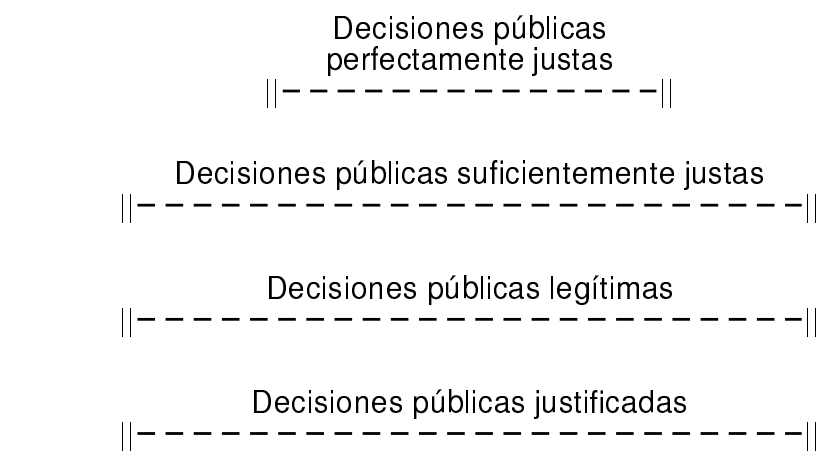

Decisiones aprobadas siguiendo procedimientos democráticos ||-- - - - - - - - - - - - - - - - - - - - - - - - - - - - |

\section{Esquema 2}

Como el esquema 2 muestra, de acuerdo con nuestro modelo de RP el dominio de las políticas suficientemente justas es coextensional con el 
dominio de las políticas públicas legítimas y las justificadas. El hecho de que tales políticas sean lo suficientemente justas $-\mathrm{y}$ no el que sean consensuadas, accesibles o aceptables por y para los ciudadanos razonables - es lo que les confiere legitimidad y las justifica. Por supuesto, como hemos visto, las decisiones suficientemente justas son aquellas que articulan de manera suficientemente plausible los valores políticos y las consideraciones afines. Quizá sea el caso que sólo una política pública de este dominio ejemplifique la justicia perfecta. Como hemos mencionado, nos parece que el desacuerdo sobre la justicia perfecta es inevitable en una sociedad democrática. Finalmente, debe notarse que el que una decisión siga procedimientos democráticos institucionalizados no necesariamente asegura que dicha decisión sea justa, legítima o justificada — aunque en una sociedad democrática toda decisión justa, legítima y justificada surja de procedimientos democráticos institucionalizados-. Este compromiso de nuestro modelo de RP nos distancia de posiciones que piensan la legitimidad en términos puramente procedimentales o puramente sustantivos.

La cuarta clarificación de nuestro modelo tiene que ver con características de las razones accesibles y aceptables que no son de contenido, sino más bien con la actitud de civilidad de quien las ofrece. Un ciudadano u oficial que da razones accesibles o aceptables, se compromete a dos cuestiones. Primero, ofrece su argumentación de forma sincera -i.e., realmente cree que las razones brindadas justifican su posición (esto lo señalan Bohman y Richarson (2009, p. 269) y Quong (2011, p. 207) - Segundo, acepta que las razones articuladas en su argumentación son siempre revisables $-\mathrm{y}$ en tal medida está dispuesto a examinarlas y modificarlas (esto lo señalan Gutmann y Thompson (2004, pp. 156-159)) - . La aceptación de estos dos compromisos, aunado al tipo de contenido de las razones accesibles y aceptables, configuran una noción de respeto ciudadano. Los ciudadanos razonables, al dar razones accesibles y aceptables siguiendo estos dos compromisos, se respetan mutuamente como iguales. Esta idea de respeto a la igualdad ciudadana es concomitante al hecho de que RP, según nuestra interpretación del triple estándar normativo, intenta preservar los términos equitativos de la cooperación social. Dichos términos se garantizan tanto de la manera en la que se conduce la discusión pública con sus respectivas restricciones como de la manera en la que se eligen ciertas políticas públicas en detrimento de otras.

Finalmente, la quinta clarificación de nuestro modelo de RP es que, a pesar de que no podemos argumentarlo a profundidad por falta de espacio, sostenemos que el triple estándar normativo de RP debe aplicarse a todas las discusiones sobre qué leyes, políticas 
públicas y decisiones adoptar. Contrariamente, Rawls (2005, pp. 262 266) sostuvo que RP era un estándar que sólo debía regular las discusiones de lo que llamó las esencias constitucionales, esto es, las cuestiones que atañen a los principios de justicia básica. Esta es una discusión sobre la amplitud de RP. Quong (2011, pp 256289) ofrece razones poderosas para defender esta idea de extender la amplitud de RP a toda discusión pública. Nosotros queremos añadir dos en función de nuestro modelo. Primero, como la cuarta clarificación sostiene, RP recrea una noción de respeto a la mutua igualdad. Segundo, como la segunda clarificación sostiene, RP no intenta dirimir todos los desacuerdos sobre cuestiones de justicia sustantiva. Una vez considerado esto, la idea de limitar su amplitud pierde una de las razones más fuertes a su favor.

No nos queda más que concluir enfatizando las tres cuestiones que nos parecen más relevantes para las discusiones contemporáneas sobre RP. Primero, cualquier modelo de RP tiene que adoptar la tesis deflacionista para evitar caer en petición de principio o en la trivialidad. Segundo, la tesis del triple estándar muestra que el objetivo final de un modelo de RP debe ser ayudar a identificar las razones correctas (en nuestra terminología, las razones aceptables) de entre aquellas que articulan valores políticos (en nuestra terminología, las razones accesibles). Esto contrasta radicalmente con los enfoques actuales en esta literatura. Finalmente, debe resaltarse la coextensionalidad del dominio de políticas públicas suficientemente justas, legítimas y justificadas. Lo primero, según nuestro modelo, determina lo segundo y lo tercero.

\section{BIBLIOGRAFÍA}

Barry, B., 1995, "John Rawls and the Search for Stability", Ethics, vol. 105, no. 4, pp. 874-915.

Berer, M., 2006, "Editorial: Condoms, Yes! 'Abstinence', No", Reproductive Health Matters, vol. 14, no. 28, pp. 6-16.

Bohman, J. y H. Richardson, 2009, "Liberalism, Deliberative Democracy, and 'Reasons that All Can Accept'", The Journal of Political Philosophy, vol. 17, no. 3, pp. 253-274.

Brettschneider, C., 2012, When the State Speaks, What Should It Say?, Princeton University Press, Princeton, Nueva Jersey.

Carter, S., 1987, "Evolutionism, Creationism, and Treating Religion as a Hobby", Yale Law School Legal Scholarship Repository, Faculty Scholarship Series, no. 2234. 
Clarke, E.H., 1873, Sex in Education; or, A Fair Chance for Girls. < http://www.gutenberg.org/files/18504/18504-h/18504-h.htm> [fecha de la última consulta $01 / 10 / 2014]$.

Cohen, J., 2009, "Truth and Public Reason", Philosophy \& Public Affairs, vol. 37, no. 1, pp. 2-42.

D’Agostino, F., 1996, Free Public Reason: Making It Up as We Go, Oxford University Press, Oxford.

DeStefano, F. et al. 2006, "Increasing Exposure to Antibody-Stimulating Proteins and Polysaccharides in Vaccines Is Not Associated with Risk of Autism", Journal of Pediatrics, vol. 163, no. 2, pp. 561-567.

Elster, J., 2004, Closing the Books, Transitional Theory in Historical Perspective, Cambridge University Press, Cambridge.

Enoch, D., 2013, “The Disorder of Public Reason", Ethics, vol. 124, no. 1, pp. 141-176.

Estlund, D., 2008, Democratic Authority. A Philosophical Framework, Princeton University Press, Princeton.

Eysenck, H.J., 2004, Decline \& Fall of the Freudian Empire, Transaction Publishers, New Brunswick.

Freeman, S., 2007, Justice and the Social Contract: Essays on Rawlsian Political Philosophy, Oxford University Press, Oxford.

Friedman, M., 2002, Capitalism and Freedom: Fortieth Anniversary Edition, University of Chicago Press, Chicago.

Gaus, G., 1996, Justifying Liberalism: An Essay on Epistemology and Political Theory, Oxford University Press, Nueva York.

George, R.P., 2001, In Defense of Natural Law, Oxford University Press, Nueva York.

—_, 2000, "Natural Law and Public Reason", en P. George y C. Wolfe 2000, pp. 51-74.

George, R.P. y C. Wolfe (comps.), 2000, Natural Law and Public Reason, Georgetown University Press, Washington.

Greenawalt, K., 1988, Religious Convictions and Political Choice, Oxford University Press, Nueva York.

Gutmann, A. y D. Thompson, 2004, Why Deliberative Democracy?, Princeton University Press, Princeton.

Cambridge, Mass.

Klosko, G., 2000, Democratic Procedures and Liberal Consensus, Oxford University Press, Nueva York.

Larmore, C., 1999, "The Moral Basis of Political Liberalism", Journal of Philosophy, vol. 96, no. 12, pp. 599-625.

Leland, R. y H. Wietmarschen, 2012, "Reasonableness, Intellectual Modesty, and Reciprocity in Political Justification", Ethics, vol. 122, no. 4, pp. 721-747.

Macedo, S., 2000, "In Defense of Liberal Public Reason: Are Abortion and Slavery Hard Cases?", en R.P. George y C. Wolfe (comps.), pp. 11-49. 
Nagel, T., 1987, "Moral Conflict and Political Legitimacy", Philosophy and Public Affairs, vol. 16, no. 3 pp. 215-240.

Quinn, P., 1995, "Political Liberalism and Their Exclusions of the Religious", Proceedings and Addresses of the American Philosophical Association, vol. 69, no. 2, pp. 35-56.

Quong, J., 2011, Liberalism without Perfection, Oxford University Press, Nueva York.

Rawls J., 2005, Political Liberalism, Columbia University Press, Nueva York. [Versión en castellano: Liberalismo político, trad. Antoni Domènech, $1^{\mathrm{a}}$ reimpresión, Crítica, Barcelona, 2006.]

- 1999, The Idea of Public Reason Revisited, en "Collected Papers", Harvard University Press, Cambridge, Mass. [Versión en castellano: El derecho de gentes y "Una revisión de la idea de razón pública", trad. Hernando Valencia Villa, Paidós, Estado y Sociedad, Barcelona, 2001.]

Reidy, D.A., 2000, "Rawls's Wide View of Public Reason: Not Wide Enough", Res Publica, vol. 6, no. 1, pp. 49-72.

Robcis, C., 2013, The Law of Kinship: Anthropology, Psychoanalysis, and the Family in Twentieth Century France, Cornell University Press, Nueva York.

Rogel H., 1977, "El comienzo de una vida humana", libro anual del ISEE (Instituto Superior de Estudios Eclesiásticos), vol. VI, México, D.F.

Sandel, M.J., 1998, Liberalism and the Limits of Justice, Cambridge University Press, Nueva York.

Schwartzmann, M., 2004, "The Completeness of Public Reason", Politics, Philosophy \& Economics, vol. 3, no. 2, pp. 19-220.

Simmons, J., 2001, Justification and Legitimacy. Essays on Rights and Obligation, Cambridge University Press, Cambridge.

- , 1999, "Justification and Legitimacy", Ethics, vol. 109, no. 4, pp. 739-771.

Tooley, M., 1984, "In Defense of Abortion and Infanticide", en Joel Feinberg (comp.), The Problem of Abortion, The University of Arizona, Belmont.

Vallier, K., 2011, “Against Public Reason Liberalism's Accessibility Requirement", Journal of Moral Philosophy, vol. 8, no. 3, pp. 366-389.

Waldron, J., 1993, "Religious Contributions in Public Deliberation", San Diego Law Review, no. 30, pp. 817-850.

Wenar, L., 2004, “The Unity of Rawls's Work", Journal of Moral Philosophy, vol. 1, no. 3, pp. 265-275.

— 1995, "Political Liberalism: An Internal Critique", Ethics, vol. 106, no. 1, pp. 32-62.

Williams, A., 2000, “The Alleged Incompleteness of Public Reason", Res Publica, vol. 6, no. 2, pp. 199-211.

Recibido el 11 de abril de 2014; revisado el 23 de septiembre de 2014; aceptado el 29 de septiembre de 2014. 